\title{
Triton $/{ }^{3} \mathrm{He}$ ratio as an observable for neutron skin thickness
}

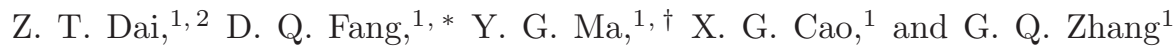 \\ ${ }^{1}$ Shanghai Institute of Applied Physics, Chinese Academy of Sciences, Shanghai 201800, China \\ ${ }^{2}$ University of Chinese Academy of Sciences, Beijing 100049, China
}

(Dated: October 24, 2018)

\begin{abstract}
Based on the framework of the Isospin-Dependent Quantum Molecular Dynamics (IQMD) model in which the initial neutron and proton densities are sampled according to the droplet model, the correlation between triton-to- ${ }^{3} \mathrm{He}$ yield ratio $\left(\mathrm{R}\left(\mathrm{t} /{ }^{3} \mathrm{He}\right)=\mathrm{Yield}(\mathrm{t}) / \mathrm{Yield}\left({ }^{3} \mathrm{He}\right)\right)$ and neutron skin thickness $\left(\delta_{n p}\right)$ in neutron-rich projectile induced reactions is investigated. By changing the diffuseness parameter of neutron density distribution in the droplet model for the projectile to obtain different $\delta_{n p}$, the relationship between $\delta_{n p}$ and the corresponding $\mathrm{R}\left(\mathrm{t} /{ }^{3} \mathrm{He}\right)$ in semi-peripheral collisions is obtained. The calculated results show that $\mathrm{R}\left(\mathrm{t} /{ }^{3} \mathrm{He}\right)$ has a strong linear correlation with $\delta_{n p}$ for neutron-rich ${ }^{50} \mathrm{Ca}$ and ${ }^{68} \mathrm{Ni}$ nuclei. It is suggested that $\mathrm{R}\left(\mathrm{t} /{ }^{3} \mathrm{He}\right)$ could be regarded as a good experimental observable to extract $\delta_{n p}$ for neutron-rich nuclei because the yields of charged particles triton and ${ }^{3} \mathrm{He}$ can be measured quite precisely.
\end{abstract}

PACS numbers: 21.10.Gv, 24.10.-i, 25.70.Mn

\section{INTRODUCTION}

The proton and neutron density distributions are some of the most fundamental properties of nuclei. Charge radii of nuclei can be derived from charge density distributions which can be determined to a high accuracy (often with the accuracy in charge radii better than 1\% or better for many nuclei) by experiments using electromagnetic probes, for example, electron scattering experiments 1]. The empirical information of proton radii is then obtained from these charge radii. In contrast, our knowledge of neutron distributions, which have been studied mainly by hadron-nucleus scattering, is limited because the descriptions of strong interactions in nuclei are highly model dependent [2]. Reliable neutron distributions will improve our understanding of the nucleus and nuclear matter [3 6$]$.

Nuclei with neutron number $(N)$ larger than proton number $(Z)$ are expected to have a neutron skin. The skin thickness is defined as the difference between the neutron and proton root-mean-square (rms) radii: $\delta_{n p}=\left\langle r_{n}^{2}\right\rangle^{1 / 2}-\left\langle r_{p}^{2}\right\rangle^{1 / 2}$. The neutron skin thickness depends on the balance between various aspects of the nuclear force. The formation of neutron skin arises because of the large neutron excess and also the difference of potentials for neutron and proton [7]. Strong correlations between $\delta_{n p}$ and $E_{s y m}\left(\rho_{0}\right)$ (nuclear symmetry energy at saturation density $\rho_{0}$ ), $L$ (the slope of symmetry energy ), $K_{\text {sym }}$ ( the curvature of the nuclear symmetry energy at $\left.\rho_{0}\right)$, the ratio $L / J(J$ is the symmetry energy coefficient at the saturation density $\left.\rho_{0}\right), \mathrm{J}-a_{\text {sym }}\left(a_{\text {sym }}\right.$ is the symmetry energy coefficient of finite nuclei) have been demonstrated [3, 8 -13]. These constraints are important for extrapolation of the nuclear equation of state (EOS)

\footnotetext{
*Email: dqfang@sinap.ac.cn

${ }^{\dagger}$ Email: ygma@sinap.ac.cn
}

to high density and hence useful for studying properties of neutron star. Moreover, a large number of correlations between $\delta_{n p}$ and several neutron star quantities have also been found, such as (a) the pressure of pure neutron matter near saturation density [3, 14], (b) neutron star radii 5, 9, 15], (c) the crust-to-core transition density [8, 16], and (d) the crustal moment of inertia [11, 17]. Neutron skin thickness is also closely related with the derivation of volume and surface symmetry energy, as well as nuclear incompressibility with respective to density. Furthermore, neutron skin thickness helps to identify a nucleus with exotic structure. Thus the precise determination of neutron skin thickness for a nucleus becomes an important research subject in nuclear physics.

Several attempts have been made to determine neutron skin thickness. These include hadron scattering [18, 19], $\pi^{-}$scattering [20], antiprotonic atoms method 21, 22], giant dipole resonance (GDR) method [23, 24], spindipole resonance (SDR) method 25, 26], Gamow-Teller resonance (GTR) method [27] etc. Almost all these methods are strongly model dependent due to the complexity of the strong interaction between nucleons. And significant differences exist between these experimental results. The $\mathrm{Pb}$ radius experiment (PREX) at Jefferson Laboratory, has initiated a new line of research based on the parity-violating elastic electron scattering to measure the neutron density radius 28]. Although parityviolating elastic electron scattering provides a model independent measurement of neutron distributions, its current precision is far from satisfactory and the method cannot be applied to unstable isotopes.

By using the Isospin-Dependent Quantum Molecular Dynamics (IQMD) model, Sun et al. have investigated the neutron to proton ratio $R(n / p)$ of emitted nucleons from projectile with different neutron skin thickness and shown that there is a strong linear relationship between $R(n / p)$ and $\delta_{n p}$, especially for peripheral collisions [29]. $R(n / p)$ is proposed as a possible observable for extracting neutron skin size. However, it is quite difficult to mea- 
sure precisely the $n / p$ ratio experimentally due to the low detection efficiency for neutrons. But it is relatively much easier to measure light charged particles. Under the coalescence picture for cluster formation, the ratio of triton to ${ }^{3} \mathrm{He}\left(\mathrm{R}\left(\mathrm{t} /{ }^{3} \mathrm{He}\right)\right)$ is expected to be proportional to the $n / p$ ratio. And $\mathrm{R}\left(\mathrm{t} /{ }^{3} \mathrm{He}\right)$ can easily be measured since triton and ${ }^{3} \mathrm{He}$ are charged particles. Meanwhile, the ratio $\mathrm{R}\left(\mathrm{t} /{ }^{3} \mathrm{He}\right)$ is also found related with nuclear symmetry energy of neutron-rich nuclei [30, 31]. This ratio is also proposed as a possible observable to probe the thermodynamic properties of the fragmenting system 32 35]. Thus the ratio of triton to ${ }^{3} \mathrm{He}$ is an interesting and important physics quantity in nuclear physics. In this paper, we will explore the relationship between the triton-to- ${ }^{3} \mathrm{He}$ yield ratio $\mathrm{R}\left(\mathrm{t} /{ }^{3} \mathrm{He}\right)$ and the neutron skin thickness within the framework of IQMD model. The possibility of extracting the neutron skin thickness for neutron-rich nuclei from measurement of $\mathrm{R}\left(\mathrm{t} /{ }^{3} \mathrm{He}\right)$ will be investigated.

The paper is organized as follows. In Sec. II we briefly describe the IQMD model, the droplet model as well as the initialization of projectile and target. In Sec. III we present the correlation between the neutron skin thickness and triton-to- ${ }^{3} \mathrm{He}$ yield ratio. Finally, a summary is given in Sec. IV.

\section{THE FRAMEWORK DESCRIPTION}

The quantum-molecular-dynamics (QMD) approach is a many-body theory to describe heavy ion collisions from intermediate to relativistic energies [36, 37]. The main advantage of the QMD model is that it can explicitly treat the many-body state of the collision system and contains correlation effects for all orders. Therefore, the QMD model can provide valuable information about both the collision dynamics and the fragmentation process. It mainly consists of several parts: initialization of the projectile and the target nucleons, propagation of nucleons in the effective potential, two body nucleonnucleon (NN) collisions in a nuclear medium and the Pauli blocking.

The IQMD model is based on the general QMD model with explicitly inclusion of isospin degrees of freedom in the mean field, two-body NN collisions and the Pauli blocking. In addition, it is also important that, in initialization of the projectile and target nuclei, the samples of neutrons and protons in phase space should be treated separately since there exists a large difference between neutron and proton density distributions for nuclei far from the $\beta$-stability line. Particularly, for neutronrich nucleus one should sample a stable initialized nucleus with neutron-skin and therefore one can directly explore the nuclear structure effects through a microscopic transport model. QMD model has been widely and successfully used in heavy ion collisions. These include nuclear structure [29, 38 40], particle and fragment production [41 47], nuclear EOS [48 50], collective flow 51 54] and other subjects [55 57].

In the IQMD model, the wave function for each nucleon is represented by a Gaussian wave packet,

$\psi_{i}\left(\vec{r}, t_{i}\right)=\frac{2}{(2 \pi L)^{3 / 4}} \exp \left[-\frac{\left(\vec{r}-\overrightarrow{r_{i}}(t)\right)^{2}}{4 L}\right] \exp \left[\frac{i \vec{r} \cdot \overrightarrow{p_{i}}(t)}{\hbar}\right]$,

where $\overrightarrow{r_{i}}(t)$ and $\overrightarrow{p_{i}}(t)$ are the $i$ th wave pocket in the coordinate and momentum space. $L$ is the width of the wave pocket, which is system-size-dependent [37, 52, 58]. $L=2.16 \mathrm{fm}^{2}$ is used in the present study. And all nucleons interact via mean field and NN collisions. The nuclear mean field can be parameterized by

$$
\begin{aligned}
U\left(\rho, \tau_{z}\right)=\alpha & \left(\frac{\rho}{\rho_{0}}\right)+\beta\left(\frac{\rho}{\rho_{0}}\right)^{\gamma}+\frac{1}{2}\left(1-\tau_{z}\right) V_{c} \\
& +C_{\text {sym }} \frac{\rho_{n}-\rho_{p}}{\rho_{0}} \tau_{z}+U^{Y u k}
\end{aligned}
$$

with $\rho_{0}=0.16 \mathrm{fm}^{-3}$ (the normal nuclear matter density). $\rho, \rho_{n}$, and $\rho_{p}$ are the total, neutron, and proton densities, respectively. $\tau_{z}$ is the $z$-th component of the isospin degree of freedom, which equals 1 or -1 for neutrons or protons, respectively. The coefficients $\alpha, \beta$ and $\gamma$ are parameters of the nuclear EOS. $C_{\text {sym }}$ is the symmetry energy strength due to the difference between neutron and proton asymmetry in nuclei, which takes the value of $32 \mathrm{MeV}$. In this paper, $\alpha=-356 \mathrm{MeV}, \beta=303 \mathrm{MeV}$ and $\gamma=7 / 6$ are taken, which corresponds to the socalled soft EOS. $V_{c}$ is the Coulomb potential and $U^{Y u k}$ is Yukawa (surface) potential.

In the phase space initialization of the projectile and target, the density distributions of proton and neutron are distinguished from each other. The neutron and proton densities for the initial projectile and target nuclei in the present IQMD model are taken from the droplet model. In the droplet model [59, 60], we can change the diffuseness parameter to get different skin size in density distributions,

$$
\rho_{i}(r)=\frac{\rho_{i}^{0}}{1+\exp \left(\frac{r-C_{i}}{f_{i} t_{i} / 4.4}\right)}, \quad i=n, p,
$$

where $\rho_{i}^{0}$ is the normalization constant which ensures that the integration of the density distribution equals to the number of neutrons $(i=\mathrm{n})$ or protons $(i=\mathrm{p}) ; t_{i}$ is the diffuseness parameter; $C_{i}$ is the half density radius of neutron or proton determined by the droplet model [60]

$$
C_{i}=R_{i}\left[1-\left(b_{i} / R_{i}\right)^{2}\right], \quad i=n, p .
$$

Here $b_{i}=0.413 f_{i} t_{i}, R_{i}$ is the equivalent sharp surface radius of neutron or proton. $R_{i}$ and $t_{i}$ are given by the droplet model. In Ref. 22], Trzcińska et al. found that the half density radii for neutrons and protons in heavy nuclei are almost the same, but the diffuseness parameter for neutron is larger than that for the proton which determines the neutron skin thickness. Especially for neutronrich nuclei far from the stability line, a large neutron skin 
is expected. Therefore, a factor $f_{i}$ is introduced by us to adjust the diffuseness parameter. In the calculation for neutron-rich nucleus, $f_{p}=1.0$ is used in Eq. (3) for the proton density distribution, while $f_{n}$ in Eq. (3) is changed from 1.0 to 1.5. Different values of $\delta_{n p}$ can be deduced from Eq.(3) with different $f_{n}$ values. Using the density distributions given by the droplet model, we can get the initial coordinate of nucleons in the nucleus in terms of the Monte Carlo sampling method. In the IQMD model, the nucleon radial density can be written as:

$$
\begin{aligned}
\rho(r)= & \sum_{i} \frac{1}{(2 \pi L)^{3 / 2}} \exp \left(-\frac{r^{2}+r_{i}^{2}}{2 L}\right) \frac{L}{2 r r_{i}} \\
& \times\left[\exp \left(\frac{r r_{i}}{L}\right)-\exp \left(-\frac{r r_{i}}{L}\right)\right],
\end{aligned}
$$

with the summation over all nucleons. And the momentum distribution of nucleons is generated by means of the local Fermi gas approximation. The Fermi momentum is calculated by

$$
P_{F}^{i}(\overrightarrow{\mathbf{r}})=\hbar\left[3 \pi^{3} \rho_{i}(\overrightarrow{\mathbf{r}})\right]^{1 / 3} \quad i=n, p .
$$

To avoid taking an unstable initialization of projectile and target in the IQMD calculation, the stability of the sampled nuclei is strictly checked by the time evolution in the mean field until $200 \mathrm{fm} / \mathrm{c}$ at zero temperature according to the average binding energies, rms radii, and density distributions of the neutrons and protons. In the initialization, the projectile and target nucleus are treated differently. Eligible initialization samples of projectile should meet the following requirements until 200 $\mathrm{fm} / \mathrm{c}$ : (i) The average binding energy matches with the experimental data; (ii) the rms radius is in accordance with the droplet model; (iii) the neutron skin thickness of projectile averaged on time is consistent with the droplet model. While the target ${ }^{12} \mathrm{C}$ only have to keep stable in the evolution time. Using the selected initialization phase space of nuclei in IQMD to simulate the collisions, the nuclear fragments are constructed by a coalescence model, in which nucleons with relative momentum smaller than $\mathrm{P}_{0}=300 \mathrm{MeV} / \mathrm{c}$ and relative distance smaller than $\mathrm{R}_{0}$ $=3.5 \mathrm{fm}$ will be combined into a cluster. And there are many different methods of clusterization [30, 47, 61, 62]. Different clustering methods may change the production rate of fragments, but the ratio $\mathrm{R}\left(\mathrm{t} /{ }^{3} \mathrm{He}\right)$ is less modeldependent and also less affected by other effects [30].

\section{RESULTS AND DISCUSSIONS}

The semi-peripheral collision processes of ${ }^{50} \mathrm{Ca}$ and ${ }^{68} \mathrm{Ni}$ with ${ }^{12} \mathrm{C}$ target at $50 \mathrm{MeV} /$ nucleon are simulated using the IQMD model. The relationship between tritonto- ${ }^{3} \mathrm{He}$ yield ratio and the neutron skin thickness in the projectile is investigated. The fragments including neutrons and protons that formed during the evolution of the collision are constructed by the coalescence method. The

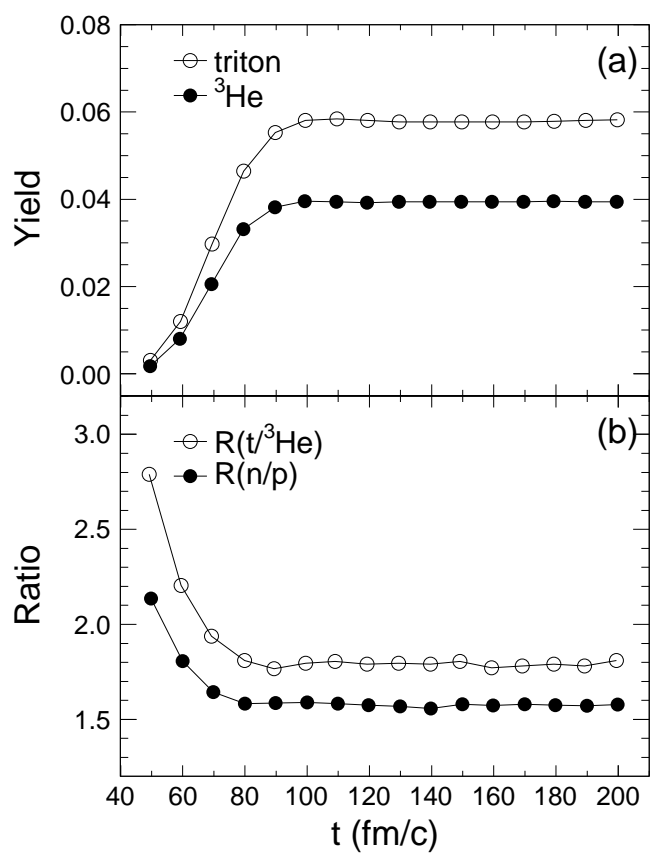

FIG. 1: (a) Time evolution of the yield (per event) for triton and ${ }^{3} \mathrm{He}$; (b) time evolution of $\mathrm{R}(\mathrm{n} / \mathrm{p})$ and $\mathrm{R}\left(\mathrm{t} /{ }^{3} \mathrm{He}\right)$ for ${ }^{68} \mathrm{Ni}+{ }^{12} \mathrm{C}$ at $50 \mathrm{MeV} /$ nucleon under the condition of reduced impact parameter from 0.6 to 1.0 and $y>0$.

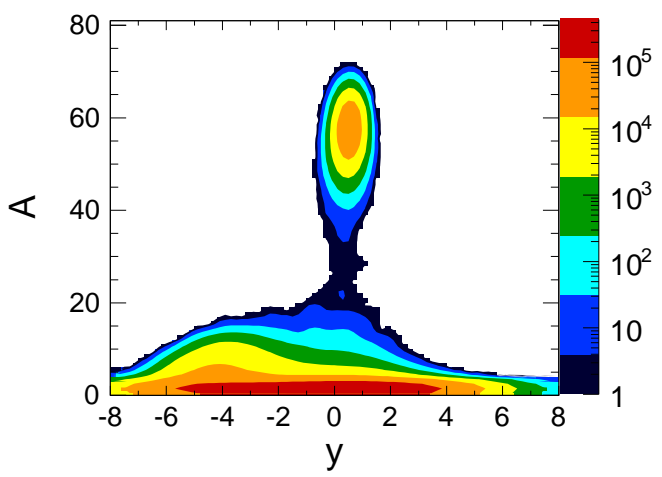

FIG. 2: (Color online) Mass number versus normalized rapidity of the produced fragments for ${ }^{68} \mathrm{Ni}+{ }^{12} \mathrm{C}$ at 50 $\mathrm{MeV} /$ nucleon under the condition of reduced impact parameter from 0.6 to 1.0 and the evolution time from $150 \mathrm{fm} / \mathrm{c}$ to $200 \mathrm{fm} / \mathrm{c}$.

yield ratio $\mathrm{R}(\mathrm{n} / \mathrm{p})$ and $\mathrm{R}\left(\mathrm{t} /{ }^{3} \mathrm{He}\right)$ can be calculated from the emitted neutrons, protons, tritons and ${ }^{3} \mathrm{He}$. It is assumed that the emitted fragments will have a memory of the $N / Z$ of the quasiprojectile in peripheral collisions. As the neutron skin thickness increases, the neutronproton composition in the surface of nucleus will also increase. Thus the yield ratio $\mathrm{R}\left(\mathrm{t} /{ }^{3} \mathrm{He}\right)$ will carry the initial neutron-proton composition $(N / Z)$ of its emitting source. By changing the factor $f_{n}$ in the neutron density distribution of the droplet model for the projectile, 


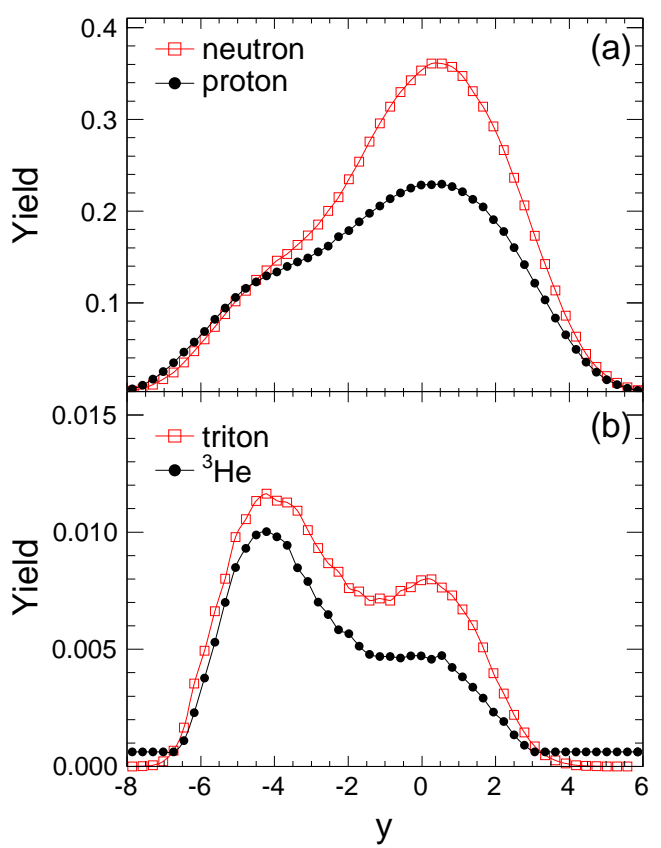

FIG. 3: (Color online) (a) The normalized rapidity distribution of neutron and proton; (b) the normalized rapidity distribution of triton and ${ }^{3} \mathrm{He}$ for ${ }^{68} \mathrm{Ni}+{ }^{12} \mathrm{C}$ at $50 \mathrm{MeV} /$ nucleon. The calculation condition is the same as Fig. 2

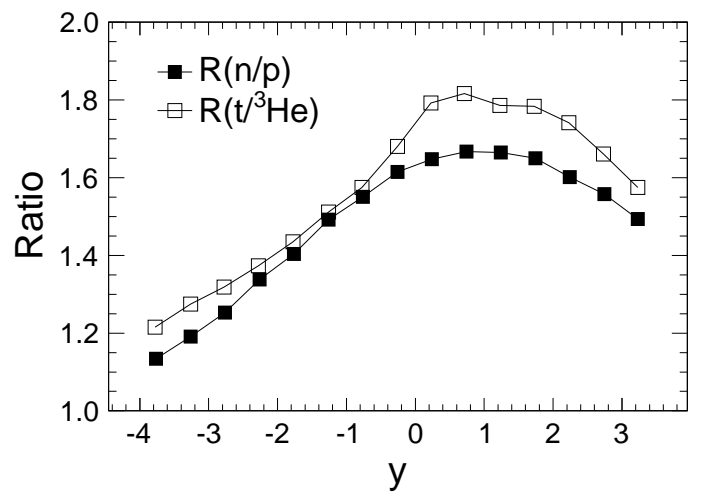

FIG. 4: $\mathrm{R}(\mathrm{n} / \mathrm{p})$ and $\mathrm{R}\left(\mathrm{t} /{ }^{3} \mathrm{He}\right)$ as a function of normalized rapidity $y$ for ${ }^{68} \mathrm{Ni}+{ }^{12} \mathrm{C}$ at $50 \mathrm{MeV} /$ nucleon. The calculation condition is the same as Fig. 2 .

different values of $\delta_{n p}$ and the corresponding $\mathrm{R}(\mathrm{n} / \mathrm{p})$ and $\mathrm{R}\left(\mathrm{t} /{ }^{3} \mathrm{He}\right)$ are obtained. Consequently, we can obtain the correlation between $\mathrm{R}(\mathrm{n} / \mathrm{p}), \mathrm{R}\left(\mathrm{t} /{ }^{3} \mathrm{He}\right)$ and $\delta_{n p}$.

Since the main purpose of the present work is to study the effect of neutron skin thickness of the projectile on the yield ratio of emitted particles. The calculation will focus on the production of fragment from the projectile in semi-peripheral collision. The reduced impact parameter is used to describe the centrality of collision which is defined as $b / b_{\max }$ with $b_{\max }$ being the maximum impact parameter. Since the main difference between neutron and proton density distribution is in the surface of nu- cleus, the probe $\mathrm{R}\left(\mathrm{t} /{ }^{3} \mathrm{He}\right)$ may be much more sensitive in peripheral collisions. While the statistics also need to be taken into account. Thus $0.6<b / b_{\max }<1.0$ is used in the calculation. In order to minimize the target effect on $\mathrm{R}(\mathrm{n} / \mathrm{p})$ and $\mathrm{R}\left(\mathrm{t} /{ }^{3} \mathrm{He}\right)$, we use rapidity $(y)$ cut to select neutrons, protons, tritons and ${ }^{3} \mathrm{He}$ emitted from the projectile. The rapidity of the fragments normalized to the incident projectile rapidity is defined as:

$$
y=\frac{1}{2} \ln \left(\frac{E+p_{z}}{E-p_{z}}\right) / y_{\text {proj }},
$$

where $E$ is the energy of the fragment, $p_{z}$ is the momentum of fragment in $z$ direction and $y_{\text {proj }}$ is the rapidity of the projectile. All calculations are carried out in the center-of-mass system (CMS).

In the calculation, the time evolution of the dynamical process was simulated until $\mathrm{t}=200 \mathrm{fm} / \mathrm{c}$. As shown in Fig. 11, the yields of produced tritons and ${ }^{3} \mathrm{He}$ (upper panel), together with the corresponding $\mathrm{R}\left(\mathrm{t} /{ }^{3} \mathrm{He}\right)$ and also R(n/p) (lower panel) are stable after $100 \mathrm{fm} / \mathrm{c}$. From Fig. 1(b), one can see that $\mathrm{R}\left(\mathrm{t} /{ }^{3} \mathrm{He}\right)$ is larger than $\mathrm{R}(\mathrm{n} / \mathrm{p})$ throughout the whole evolution process. The reason may be that it is easier to combine a neutron into a cluster than a proton due to the Coulomb repulsion as well as the neutron-rich environment. In order to improve statistics we accumulate the emitted neutrons, protons, tritons and ${ }^{3} \mathrm{He}$ between $150 \mathrm{fm} / \mathrm{c}$ and $200 \mathrm{fm} / \mathrm{c}$.

While analyzing the rapidity distribution of different fragments in semi-peripheral collisions, an interesting phenomenon was found as shown in Fig. 2. For a certain kind of light cluster, there are more particles distributing in the target-like region rather than in the projectile-like region, which is contrary to our expectation. The rapidity distribution of neutrons, protons, tritons and ${ }^{3} \mathrm{He}$ are shown in Fig. [3 respectively. From the figure one can see that there are more neutrons and protons in projectilelike region, while for tritons and ${ }^{3} \mathrm{He}$ it is opposite. To better understand what happened in the collision process, we followed the tracks of the nucleons in the whole collision process. It turns out that the ${ }^{12} \mathrm{C}$ target is much easier to break up than the neutron-rich projectiles ${ }^{50} \mathrm{Ca}$ and ${ }^{68} \mathrm{Ni}$. This can be explained as a geometry effect. In semi-peripheral collision of such an asymmetry system, the target is almost penetrated by nucleons from the projectile since the size of the projectile is much larger than the target. While only a limited part of nucleons in the projectile is abraded.

The rapidity distribution of $\mathrm{R}(\mathrm{n} / \mathrm{p})$ and $\mathrm{R}\left(\mathrm{t} /{ }^{3} \mathrm{He}\right)$ are plotted in Fig. 目 It is interesting to see that the ratio corresponding to $A=3$ clusters indeed displays a similar rapidity dependence to that of the emitted neutrons and protons, though opposite rapidity dependence in their yields as discussed above. From this figure, we can see that $\mathrm{R}(\mathrm{n} / \mathrm{p})$ and $\mathrm{R}\left(\mathrm{t} /{ }^{3} \mathrm{He}\right)$ in projectile-like region are much larger than that in the target-like region. This is because the neutron-rich projectile will produce more neutrons than protons. However, what we are interested in is the fragments produced from the neutron-rich pro- 


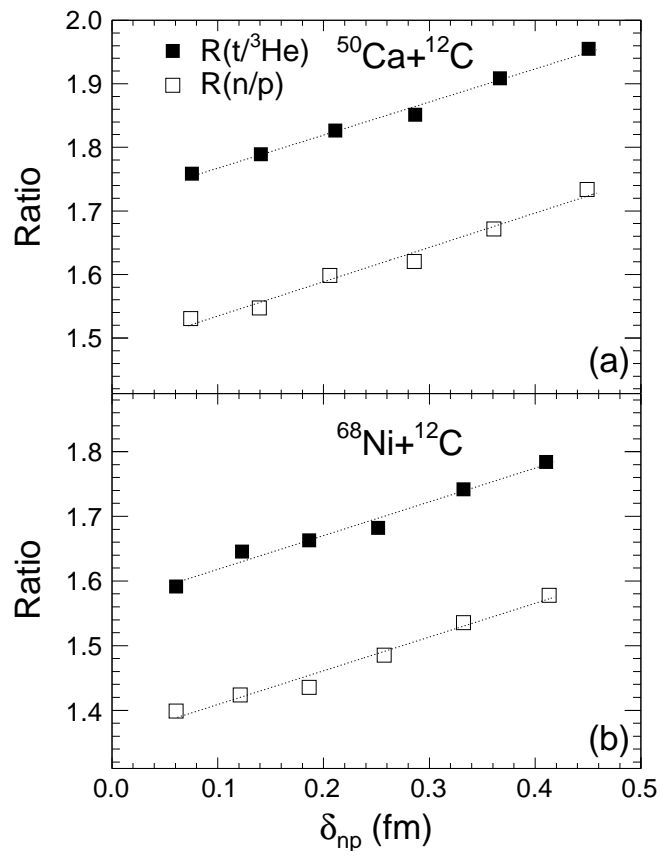

FIG. 5: Dependence of $\mathrm{R}(\mathrm{n} / \mathrm{p})$ and $\mathrm{R}\left(\mathrm{t} /{ }^{3} \mathrm{He}\right)$ on neutron skin thickness under the condition of $0.6<b / b_{\max }<1.0$ and $y>0$ for ${ }^{50} \mathrm{Ca}+{ }^{12} \mathrm{C}$ (a) and ${ }^{68} \mathrm{Ni}+{ }^{12} \mathrm{C}(\mathrm{b})$ at $50 \mathrm{MeV} /$ nucleon. The dotted lines are linear fitting.

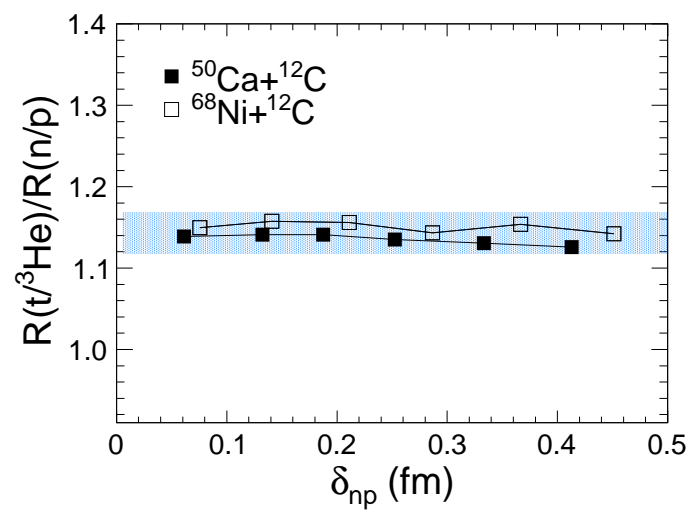

FIG. 6: (Color online) Double ratio $\mathrm{R}\left(\mathrm{t} /{ }^{3} \mathrm{He}\right) / \mathrm{R}(\mathrm{n} / \mathrm{p})$ as a function of $\delta_{n p}$ for ${ }^{50} \mathrm{Ca}+{ }^{12} \mathrm{C}$ and ${ }^{68} \mathrm{Ni}+{ }^{12} \mathrm{C}$ system at 50 $\mathrm{MeV} /$ nucleon. The calculating condition is the same as Fig. 5 The blue filled area is just to guide the eyes.

jectile, so we make a cut of rapidity $y>0$ to strip away fragments coming from the target. On the other hand, the yield ratios are much more sensitive to the surface of the nucleus, so only the events with the reduced impact parameter from 0.6 to 1.0 are taken into account. The yields of neutrons, protons, tritons and ${ }^{3} \mathrm{He}$ are selected under the condition with $0.6<b / b_{\max }<1.0$ and $y>0$ and the emitted time from $150 \mathrm{fm} / \mathrm{c}$ and $200 \mathrm{fm} / \mathrm{c}$. The corresponding ratios $\mathrm{R}\left(\mathrm{t} /{ }^{3} \mathrm{He}\right)$ and $\mathrm{R}(\mathrm{n} / \mathrm{p})$ with respect to the neutron skin thickness for ${ }^{50} \mathrm{Ca}+{ }^{12} \mathrm{C}$ and ${ }^{68} \mathrm{Ni}+{ }^{12} \mathrm{C}$ systems are plotted in Fig. 5. A strong linear correlation between $\mathrm{R}\left(\mathrm{t} /{ }^{3} \mathrm{He}\right), \mathrm{R}(\mathrm{n} / \mathrm{p})$ and $\delta_{n p}$ is exhibited, which indicates that both $\mathrm{R}\left(\mathrm{t} /{ }^{3} \mathrm{He}\right)$ and $\mathrm{R}(\mathrm{n} / \mathrm{p})$ are sensitive to $\delta_{n p}$. And $\mathrm{R}\left(\mathrm{t} /{ }^{3} \mathrm{He}\right)$ is always larger than $\mathrm{R}(\mathrm{n} / \mathrm{p})$ as mentioned previously.

The double ratios $\mathrm{R}\left(\mathrm{t} /{ }^{3} \mathrm{He}\right) / \mathrm{R}(\mathrm{n} / \mathrm{p})$ as a function of $\delta_{n p}$ for both ${ }^{50} \mathrm{Ca}+{ }^{12} \mathrm{C}$ and ${ }^{68} \mathrm{Ni}+{ }^{12} \mathrm{C}$ systems are plotted in Fig. 6. One can see that, with the increasing of the neutron skin thickness, the double ratio $\mathrm{R}\left(\mathrm{t} /{ }^{3} \mathrm{He}\right) / \mathrm{R}(\mathrm{n} / \mathrm{p})$ is almost constant, which indicates that $\mathrm{R}\left(\mathrm{t} /{ }^{3} \mathrm{He}\right)$ is proportional to the $n / p$ ratio for different $\delta_{n p}$, which is consistent with the coalescence method. Thus both $\mathrm{R}\left(\mathrm{t} /{ }^{3} \mathrm{He}\right)$ and $\mathrm{R}(\mathrm{n} / \mathrm{p})$ could be used as experimental observables for determination of the neutron skin thickness. However, $\mathrm{R}\left(\mathrm{t} /{ }^{3} \mathrm{He}\right)$ will be a better quantity from the experimental point of view since the charged particles triton and ${ }^{3} \mathrm{He}$ could be measured much easier than neutrons. To see the linear relation between $\mathrm{R}\left(\mathrm{t} /{ }^{3} \mathrm{He}\right)$ and the neutron skin thickness will be changed or not by varying the parameters in IQMD model, effect of the width of Gaussian wave packet $(L)$ is investigated. The results show that a small change in $L$ has almost no effect on $\mathrm{R}\left(\mathrm{t} /{ }^{3} \mathrm{He}\right)$ and its linear dependence on neutron skin thickness. The system of ${ }^{48} \mathrm{Ca}+{ }^{12} \mathrm{C}$ is calculated to study the effect of neutrons in the projectile, the value of $\mathrm{R}\left(\mathrm{t} /{ }^{3} \mathrm{He}\right)$ will decrease by about $7 \%$ compared with ${ }^{50} \mathrm{Ca}+{ }^{12} \mathrm{C}$ which is consistent with the change of $N / Z$ from ${ }^{50} \mathrm{Ca}$ (1.5) to ${ }^{48} \mathrm{Ca}(1.4)$. But the slope of linear dependence between $\mathrm{R}\left(\mathrm{t} /{ }^{3} \mathrm{He}\right)$ and $\delta_{n p}$ is the same. Furthermore, the effect of MDI interaction is studied. With the MDI potential, the system becomes unstable and it is very difficult to have stable initial samples compared with the EOS without MDI interaction. Further investigation is necessary for understanding the effect of MDI interaction on the relation between $\mathrm{R}\left(\mathrm{t} /{ }^{3} \mathrm{He}\right)$ and $\delta_{n p}$.

\section{SUMMARY}

Within the framework of the IQMD model in which the initial neutron and proton densities are sampled according to the droplet model, we have simulated the semiperipheral collisions of $50 \mathrm{MeV} /$ nucleon ${ }^{50} \mathrm{Ca}$ and ${ }^{68} \mathrm{Ni}$ on a ${ }^{12} \mathrm{C}$ target. Assuming different neutron skin thickness for the projectile, we have studied the correlation between triton-to- ${ }^{3} \mathrm{He}$ yield ratio and neutron skin thickness for the first time. A strong linear relationship is obtained between $\mathrm{R}\left(\mathrm{t} /{ }^{3} \mathrm{He}\right)$ and $\delta_{n p}$ for neutron-rich projectile, similar with the relationship between $R(n / p)$ and $\delta_{n p}$. Since light charged particles could be measured easily in experiment as compared with neutrons, $\mathrm{R}\left(\mathrm{t} /{ }^{3} \mathrm{He}\right)$ could be regarded as a sensitive and practical observable of $\delta_{n p}$ for neutron-rich nuclei. If the emitted tritons and ${ }^{3} \mathrm{He}$ are measured by experiment, it is possible to extract $\delta_{n p}$ from $\mathrm{R}\left(\mathrm{t} /{ }^{3} \mathrm{He}\right)$. Furthermore, some information about the nuclear equation of state could be deduced after the determination of neutron skin thickness from the experimental measurements. 


\section{Acknowledgements}

This work was supported in part by the Major State Basic Research Development Program in China under
Contract No. 2013CB834405, the National Natural Science Foundation of China under Contract No.s 11175231 and 11035009, and the Chinese Academy of Science Foundation under Grant No. KJCX2-EW-N01.
[1] I. Angeli, At. Data Nucl. Data Tables 87, 185 (2004).

[2] L. Ray, G. W. Hoffman and W. R. Coker, Phys. Rep. 212, 223 (1992).

[3] B. A. Brown, Phys. Rev. Lett. 85, 5296 (2000).

[4] J. M. Lattimer and M. Prakash, Science 304, 536 (2004).

[5] A. W. Steiner, M. Prakash, J. M. Lattimer, P. J. Ellis, Phys. Rep. 411, 325 (2005).

[6] P. Danielewicz, Nucl. Phys. A 727, 233 (2003).

[7] N. Fukunishi, T. Otsuka, I. Tanihata, Phys. Rev. C 48, 1648 (1993).

[8] C. J. Horowitz and J . Piekarewicz, Phys. Rev. Lett. 86, 5647 (2001).

[9] C. J. Horowitz and J . Piekarewicz, Phys. Rev. C 66, 055803 (2002).

[10] S. Typel and B. A. Brown, Phys. Rev. C 64, 027302 (2001).

[11] R. J. Furnstahl, Nucl. Phys. A 706, 85 (2002).

[12] S. Karataglidis, K. Amos, B . A. Brown, and P. K. Deb, Phys. Rev. C 65, 044306 (2002).

[13] M. Centelles, X. Roca-Maza, X. Viñas, and M. Warda, Rev. Lett. 102, 122502 (2009).

[14] F. J. Fattoyev and J. Piekarewicz, Phys. Rev. C 86, 015802 (2012).

[15] C. J. Horowitz and J. Piekarewicz, Phys. Rev. C 64, 062802 (2001).

[16] I. Vidaña, C. Providencia, A. Polls, and A. Rios, Phys. Rev. C 80, 045806 (2009).

[17] F. J. Fattoyev and J. Piekarewicz, Phys. Rev. C 82, 025810 (2010).

[18] G. W. Hoffmann et al., Phys. Rev. Lett. 47, 1436 (1981).

[19] V. E. Starodubsky and N. M. Hintz, Phys. Rev. C 49, 2118 (1994).

[20] T. Takahashi, PhD thesis, University of Tokyo (1995) unpublished.

[21] P. Lubinski et al., Phys. Rev. C 57, 2962 (1998).

[22] A. Trzcinska et al., Phys. Rev. Lett. 87, 082501 (2001).

[23] A. Krasznahorkay et al., Phys. Rev. Lett. 66, 1287 (1991).

[24] G. R. Satchler, Nucl. Phys. A 472, 215 (1987).

[25] A. Krasznahorkay et al., Phys. Rev. Lett. 82, 3216 (1999).

[26] W. P. Alford and B. M. Spicer, Adv. Nucl. Phys. 24, 1 (1998).

[27] D. Vretenar, N. Paar, T. Niksic, P. Ring, Phys. Rev. Lett. 91, 262502 (2003).

[28] S. Abrahamyan et al., Phys. Rev. Lett. 108, 112502 (2012).

[29] X. Y. Sun, D. Q. Fang, Y. G. Ma et al., Phys. Lett. B 682, 396 (2010).

[30] L. W. Chen, C. M. Ko, B. A. Li, Phys. Rev. C. 68, 017601 (2003).

[31] G. C. Yong, B. A. Li, L. W. Chen, X. C. Zhang, Phys. Rev. C 80, 044608 (2009).

[32] Ph. Chomaz and F. Gulminelli, Phys. Lett. B. 447, 221 (1999).
[33] J. Cibor et al., Phys. Lett. B. 473, 29 (2000).

[34] M. Veselsky et al., Phys. Lett. B. 497, 1 (2001).

[35] C. W. Ma, S. S. Wang, Y. L. Zhang, H. LWei, Phys. Rev. C 87, 034618 (2013).

[36] J. Aichelin, A. Rosenhauer, G. Peilert, H. Stöcker, W. Greiner, Phys. Rev. Lett. 58, 1926 (1987).

[37] C. Hartnack, R. K. Puri, J. Aichelin, J. Konopka, S. A. Bass, H. Stöcker, and W. Greiner, Eur. Phys. J. A 1, 151 (1998).

[38] X. G. Cao et al., Phys. Rev. C 86, 044620 (2012).

[39] J. Y. Liu et al., Phys. Lett. B 617, 24 (2005).

[40] C. Tao, Y. G. Ma et al., Phys. Rev. C 87, 014621 (2013); Nucl. Sci. Tech. 24, 030502 (2013); J. Wang et al., Nucl. Sci. Tech. 24, 030501 (2013).

[41] Y. G. Ma, W. Q. Shen, Phys. Rev. C 51, 710 (1995).

[42] V. S. Uma Maheswari, C. Fuchs, A. Faessler, Z. S. Wang, and D. S. Kosov, Phys. Rev. C 57, 922 (1998).

[43] F. S. Zhang, L. W. Chen, Z. Y. Ming, Z. Y. Zhu, Phys. Rev. C 60, 064604 (1999).

[44] V. Kaur and S. Kumar, Phys. Rev. C 81, 064610 (2010).

[45] Sanjeev Kumar, Suneel Kumar and Rajeev. K. Puri, Phys. Rev. C 81, 014601 (2010);

Sanjeev Kumar, Suneel Kumar and Rajeev. K. Puri, Phys. Rev. C 81, 014611 (2010).

[46] J. Y. Liu, J. W. Guo, Y. Z. Xing, W. Zou and X. G. Lee, Phys. Rev. C 67, 024608 (2003).

[47] Jaivir Singh and Rajeev K. Puri, Phys. Rev. C 62, 054602 (2000);

[48] M. B. Tsang, Y. X. Zhang, P. Danielewicz, M. Famiano, Z. X. Li, W. G. Lynch, and A. W. Steiner, Phys. Rev. Lett 102, 122701 (2009).

[49] Z. Q. Feng, G. M. Jin, Phys. Lett. B 683, 140 (2010).

[50] Q. F. Li, Z. X. Li, S. Soff, M. Bleicher, and H. Stöcker, Phys. Rev. C 72, 034613 (2005).

[51] Y. G. Ma, W. Q. Shen, Phys. Rev. C 51, 3256 (1995).

[52] S. Gautam, R. Chugh, A. D. Sood, R. K. Puri, Ch. Hartnack and J. Aichelin, J. Phys. G 37, 085102 (2010).

[53] S. Gautam, A. D. Sood, R. K. Puri and J. Aichelin, Phys. Rev. C 83, 014603 (2011).

[54] R. Bansal, S. Gautam, R. K. Puri and J. Aichelin, Phys. Rev. C 87, 061602(R) (2013).

[55] N. Wang, X. Z. Wu, Z. X. Li Phys. Rev. C 67, 024604 (2003).

[56] C. L. Zhou, Y. G. Ma, D. Q. Fang, G. Q. Zhang, Phys. Rev. C 88, 024604 (2013).

[57] Y. G. Ma, Y. B. Wei, et al., Phys. Rev. C 73, 014604 (2006).

[58] N. Wang, Z. X. Li, X. Z. Wu, Phys. Rev. C 65, 064608 (2002).

[59] W. D. Myers and W. J. Swiatecki, Nucl. Phys. A 336, 267 (1980).

[60] W. D. Myers, K. H. Schmidt, Nucl. Phys. A 410, 61 (1983).

[61] Supriya Goyal and Rajeev K. Puri, Phys. Rev. C 83, 047601 (2011). 
[62] S. Kumar, Y. G. Ma, Nucl. Sci. Tech. 24, 050509 (2013). 